\title{
The Large Size Telescope of the Cherenkov Telescope Array
}

AMBROSI G. ${ }^{\mathrm{a}}$, AWANE Y. ${ }^{\mathrm{b}}$, BABA H. ${ }^{\mathrm{c}}$, BAMBA A. ${ }^{\mathrm{d}}$, BARCELÓ M. ${ }^{\mathrm{e}}$, BARRES DE ALMEIDA U. ${ }^{\mathrm{f}}$, BARRIO J.A. ${ }^{\mathrm{g}}$, BLANCH BIGAS O. ${ }^{\mathrm{e}}$, BOIX J. ${ }^{\mathrm{e}}$, BRUNETTI L. ${ }^{\mathrm{h}}$, CARMONA E. ${ }^{\mathrm{i}}$, CHABANNE E. ${ }^{\mathrm{h}}$, CHIKAWA M. ${ }^{\mathrm{j}}$, COLIN P. ${ }^{\mathrm{k}}$, CORTINA J. ${ }^{\mathrm{e}}$, CONTRERAS J.L. ${ }^{\mathrm{g}}$, DAZZI F. ${ }^{1}$, DE ANGELIS A. ${ }^{\mathrm{m}}$, DELEGLISE G. ${ }^{\mathrm{h}}$, DELGADO C. ${ }^{\mathrm{i}}$, DÍAZ C. ${ }^{\mathrm{i}}$, FIASSON A. ${ }^{\mathrm{h}}$, FINK D. ${ }^{\mathrm{k}}$, FOUQUE N. ${ }^{\mathrm{h}}$, FREIXAS L. ${ }^{\mathrm{i}}$, FRUCK C. ${ }^{\mathrm{k}}$, GADOLA A. ${ }^{\mathrm{n}}$, GARCÍA R. ${ }^{\circ}$, GASCON D. ${ }^{\mathrm{p}}$, GEFFROY N. ${ }^{\text {, }}$, GIGLIETTO N. ${ }^{\mathrm{q}}$, GIORDANO F. ${ }^{\mathrm{q}}$, GRANENA F. ${ }^{\mathrm{e}}$, GUNJI S. ${ }^{\mathrm{r}}$, HAGIWARA R. ${ }^{\mathrm{r}}$, HAMER N. ${ }^{\mathrm{i}}$, HANABATA Y. ${ }^{\mathrm{s}}$, HASSAN T. ${ }^{\mathrm{g}}$, HATANAKA K. ${ }^{\mathrm{b}}$, HAUBOLD T. ${ }^{\mathrm{k}}$, HAYASHIDA M. ${ }^{\mathrm{s}}$, HERMEL R. ${ }^{\mathrm{h}}$, HERRANZ D. ${ }^{\mathrm{g}}$, HIROTANI K. ${ }^{\mathrm{s}}$, INOUE S. ${ }^{\mathrm{s}}$, INOUE Y. ${ }^{\mathrm{s}}$, IOKA K. ${ }^{\mathrm{t}}$, JABLONSKI C. ${ }^{\text {k }}$, KAGAYA M. ${ }^{\mathrm{c}}$, KATAGIRI H. ${ }^{\mathrm{c}}$, KISHIMOTO T. ${ }^{\mathrm{b}}$, KODANI K. ${ }^{\mathrm{u}}$, KOHRI K. ${ }^{\mathrm{t}}$,

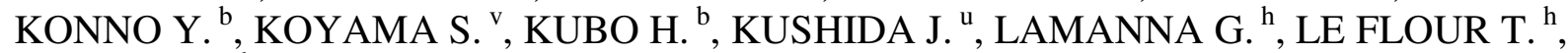
LORENZ E. ${ }^{\mathrm{k}}$, LÓPEZ R. ${ }^{\mathrm{e}}$, LÓPEZ-MOYA M. ${ }^{\mathrm{g}}$, MAJUMDAR P. ${ }^{\mathrm{w}}$, MANALAYSAY A. ${ }^{\mathrm{n}}$, MARIOTTI M. ${ }^{\mathrm{x}}$, MARTÍNEZ G. ${ }^{\mathrm{i}}$, MARTÍNEZ M. ${ }^{\mathrm{e}}$, MAZIN D. ${ }^{\mathrm{k}}$, MIRANDA J.M. ${ }^{\mathrm{y}}$, MIRZOYAN R. ${ }^{\text {, }}$, MONTEIRO I. ${ }^{\text {, }}$, MORALEJO A. ${ }^{\text {e }}$, MURASE K. ${ }^{\text {s }, ~ N A G A T A K I ~ S . ~}{ }^{\text {, }}$, NAKAJIMA D. ${ }^{\mathrm{k}}$, NAKAMORI T. ${ }^{\mathrm{aa}}$, NISHIJIMA K. ${ }^{\mathrm{u}}$, NODA K. ${ }^{\mathrm{k}}$, NOZATO A. ${ }^{\mathrm{j}}$, OHIRA Y. ${ }^{\mathrm{t}}$, OHISHI M. ${ }^{\mathrm{s}}$, OHOKA H. ${ }^{\mathrm{s}}$, OKUMURA A. ${ }^{\mathrm{bb}}$, ORITO R. ${ }^{\mathrm{cc}}$, PANAZOL J.L. ${ }^{\mathrm{h}}$, PANEQUE D. ${ }^{\mathrm{k}}$, PAOLETTI R. ${ }^{\mathrm{dd}}$, PAREDES J.M. ${ }^{\mathrm{p}}$, PAULETTA G. ${ }^{\mathrm{m}}$, PODKLADKIN S. ${ }^{\mathrm{k}}$, PRAST J. ${ }^{\mathrm{b}}$, RANDO R. ${ }^{\mathrm{x}}$, REIMANN O. ${ }^{\mathrm{k}}$, RIBÓ M. ${ }^{\mathrm{p}}$, ROSIER-LEES S. ${ }^{\mathrm{h}}$, SAITO K. ${ }^{\mathrm{s}}$, SAITO T. ${ }^{\mathrm{b}}$, SAITO Y. ${ }^{\mathrm{u}}$, SAKAKI N. ${ }^{\mathrm{s}}$, SAKONAKA R. ${ }^{\mathrm{j}}$, SANUY A. ${ }^{\mathrm{p}}$, SASAKI H. ${ }^{\mathrm{ee}}$, SAWADA M. ${ }^{\mathrm{d}}$, SCALZOTTO V. ${ }^{\mathrm{x}}$, SCHULTZ S. ${ }^{\mathrm{x}}$, SCHWEIZER T. ${ }^{\mathrm{k}}$, SHIBATA T. ${ }^{\mathrm{d}}$, SHU S. ${ }^{\mathrm{j}}$, SIEIRO J. ${ }^{\mathrm{p}}$, STAMATESCU V. ${ }^{\mathrm{e}}$, STEINER S. ${ }^{\mathrm{n}}$, STRAUMANN U. ${ }^{\mathrm{n}}$, SUGAWARA R. ${ }^{\mathrm{cc}}$, TAJIMA H. ${ }^{\text {bb }}$, TAKAMI H. ${ }^{\mathrm{t}}$, TANAKA S. $^{\mathrm{c}}$, TANAKA M. ${ }^{\mathrm{t}}$, TEJEDOR L.A. ${ }^{\mathrm{g}}$, TERADA Y. ${ }^{\mathrm{v}}$, TESHIMA M. ${ }^{\mathrm{s}, \mathrm{k}}$, TOTANI T. ${ }^{\mathrm{ff}}$, UENO H. ${ }^{\mathrm{v}}$, UMEHARA K. ${ }^{\mathrm{c}}$, VOLLHARDT A. ${ }^{\mathrm{n}}$, WAGNER R. ${ }^{\mathrm{k}}$, WETTESKIND H. ${ }^{k}$, YAMAMOTO T. ${ }^{\text {ee }}$, YAMAZAKI R. ${ }^{\mathrm{d}}$, YOSHIDA A. ${ }^{\mathrm{d}}$, YOSHIDA T. ${ }^{\mathrm{c}}$, YOSHIKOSHI T. ${ }^{\mathrm{s}}$

FOR THE CHERENKOV TELESCOPE ARRAY CONSORTIUM

a INFN Sezione di Perugia, Via A. Pascoli, Perugia, Italy

${ }^{\mathrm{b}}$ Kyoto University, Sakyo-ku, Kyoto 606-8502, Japan

${ }^{c}$ Faculty of Science, Ibaraki University, Mito, Ibaraki, 310-8512, Japan

d Department of Physics and Mathematics, Aoyama Gakuin University, Fuchinobe, Sagamihara, Kanagawa, 229-8558, Japan

e Institut de Física d'Altes Energies (IFAE), Edifici Cn, Campus UAB, 08193 Bellaterra, Spain

${ }^{\mathrm{f}}$ Centro Brasileiro de Pesquisas Fisicas (CBPF/MCTI), Rua Xavier Sigaud 150, RJ 22290-180, Rio de Janeiro, Brazil

${ }^{\mathrm{g}}$ Grupo de Altas Energías, Universidad Complutense de Madrid, Av Complutense s/n, 28040 Madrid, Spain

${ }^{\mathrm{h}}$ LAPP, Universite de Savoie, CNRS/IN2P3, 9 Chemin de Bellevue - BP 110, 74941 Annecy-leVieux Cedex, France

Ground-based and Airborne Telescopes V, edited by Larry M. Stepp, Roberto Gilmozzi, Helen J. Hall, Proc. of SPIE Vol. 9145, 91450P • (c) 2014 SPIE CCC code: $0277-786 \mathrm{X} / 14 / \$ 18 \cdot$ doi: $10.1117 / 12.2054605$ 
${ }^{\mathrm{i}}$ CIEMAT, Avda. Complutense 22, 28040 Madrid, Spain

${ }^{\mathrm{j}}$ Department of Physics, Kinki University, Kowakae, Higashi-Osaka 577-8502, Japan

${ }^{\mathrm{k}}$ Max-Planck-Institut für Physik, Föhringer Ring 6, 80805 München, Germany

${ }^{1}$ INFN Sezione di Padova, Via Marzolo 8, 35131 Padova, Italy

${ }^{m}$ University of Udine \& INFN Sezione di Trieste, Via delle Scienze 208, 33100 Udine, Italy

${ }^{\mathrm{n}}$ Physik-Institut, Universitat Zürich, Winterthurerstrasse 190, 8057 Zürich, Switzerland

${ }^{\circ}$ Instituto de Astrofísica de Canarias (IAC), Via Lactea, 38205 La Laguna, Tenerife, Spain

${ }^{\mathrm{p}}$ Departament d'Astronomia i Meteorologia (ICC-UB), Universitat de Barcelona, Martí i Franques,

1, 08028, Barcelona, Spain

${ }^{\mathrm{q}}$ INFN Sezione di Bari, via Orabona 4, I-70126 Bari, Italy

${ }^{\mathrm{r}}$ Department of Physics, Yamagata University, Yamagata, Yamagata 990-8560, Japan

s Institute for Cosmic Ray Research (ICRR), University of Tokyo, 5-1-5, Kashi-wanoha, Kashiwa, Chiba 277-8582, Japan

${ }^{\mathrm{t}}$ Institute of Particle and Nuclear Studies, KEK, 1-1 Oho, Tsukuba, 305-0801, Japan

u Department of Physics, Tokai University, 1117 Kita-Kaname, Hiratsuka, Kanagawa 259-1292,

Japan

${ }^{v}$ Saitama University, 255 Simo-Ohkubo, Sakura-ku, Saitama city, Saitama 338-8570, Japan

${ }^{w}$ Saha Institute of Nuclear Physics (SINP), Kolkata, India

${ }^{x}$ Dipartimento di Fisica, Università degli Studi di Padova, Via Marzolo 8, 35131 Padova, Italy

${ }^{y}$ Grupo de Electronica, Universidad Complutense de Madrid, Av. Complutense s/n, 28040 Madrid, Spain

${ }^{\mathrm{z}}$ RIKEN, Advanced Science Institute, 2-1 Hirosawa, Wako, Saitama 351-0198, Japan

${ }^{\text {aa }}$ Faculty of Science and Engineering, Waseda University, Shinjuku, Tokyo 169-8555, Japan

bb Nagoya University, Chikusa-ku, Nagoya 464-8602, Japan

${ }^{c c}$ Institute of Socio-Arts and Sciences, University of Tokushima, Tokushima 770-8502, Japan

dd Università di Siena \& INFN Sezione di Pisa, Via Roma 56, 53100 Siena, Italy '

ee Department of Physics, Konan University, Kobe, Hyogo, 658-8501 Japan

${ }^{\mathrm{ff}}$ Department of Physics, Graduate School of Science, University of Tokyo, 7-3-1 Hongo, Bunkyo-

ku, Tokyo 113-0033, Japan

\begin{abstract}
The Cherenkov Telescope Array (CTA) project aims to implement the world's largest next generation of Very High Energy gamma-ray Imaging Atmospheric Cherenkov Telescopes devoted to the observation from a few tens of GeV to more than $100 \mathrm{TeV}$. To view the whole sky, two CTA sites are foreseen, one for each hemisphere. The sensitivity at the lowest energy range will be dominated by four Large Size Telescopes, LSTs, located at the center of each array and designed to achieve observations of high red-shift objects with the threshold energy of $20 \mathrm{GeV}$. The LST is optimized also for transient low energy sources, such as Gamma Ray Bursts (GRB), which require fast repositioning of the telescope. The overall design and the development status of the first LST telescope will be discussed.
\end{abstract}

Keywords: Imaging Atmospheric Cherenkov Telescope, CTA, gamma-rays

\title{
1. INTRODUCTION
}

Based on the recent success of the latest generation of large Imaging Air Cherenkov Telescopes (IACTs), such as H.E.S.S., MAGIC and VERITAS, and the observation of a large number of high energy gamma-ray emitters observed by satellite borne detectors, AGILE and Fermi, it has been proposed to build an array of many IACTs of three different sizes 
( $23 \mathrm{~m}, 12 \mathrm{~m}$ and $6 \mathrm{~m}$ diameter) to achieve a sensitivity which is at least 10 times higher than that of current most powerful instruments. The detector complex is currently dubbed Cherenkov Telescope Array (CTA) [1]. In order to cope with the rapidly dropping fluxes one needs many telescopes spread over a very large area. In its current phase of planning, CTA is optimized to cover the energy range from $20 \mathrm{GeV}$ to about $100 \mathrm{TeV}$; the lower energy range in the array will be covered by four to five large size telescope, the mid-energy range by 20 to 30 mid-size telescopes and coverage for the highest energies will be given by at 50 to 60 small size telescopes.

\section{THE DESIGN OF THE LARGE SIZE TELESCOPE}

The design concept of the Large Size Telescope (LST) follows the IACT proven design of the successfully operating MAGIC telescopes but with the emphasis on an almost twice larger mirror, lower weight and use of advanced solutions and components for reducing costs and maintenance, and increasing remote operability. Figure 1 shows a rendered picture of the LST design.

The telescope is based on an alt-azimuth design, with a focal length of $28 \mathrm{~m}$ and an increased ratio $\mathrm{f} / \mathrm{D}=1.2$. The stiffness of the dish and also the camera supporting arch is achieved by the use of carbon fiber ropes that fix the arch to the dish and also from the backside of the dish to the backside arch, where the elevation-drive chain is fastened. Using ropes instead of thick tubes reduces the shadowing effect of the dish.

An access tower will allow access to the camera when the telescope is in its park position during day-time as well as acting as an anchor during strong storms.

The telescope has been designed to withstand storms of up to $200 \mathrm{Km} / \mathrm{h}$ and strong earthquakes as they can happen in some of the proposed sites such as Chile. The telescope must also hold a significant snow and ice load.

The LST is divided in three main assemblies:

1. Mechanical System: it contains the mechanical structures and the civil constructions of the telescope.

2. Optical System: it includes the reflective surface and the electro-mechanical system, so called AMC, which makes the optics adaptive.

3. Camera: it is a close case that incorporates the photo-sensors, the front end electronics and the readout. The rest of the system is made by auxiliary systems that comprehend all the remaining items that are not mentioned in the previous assemblies, as for instance the energy storage, the pointing system, the calibration system, etc. They will not be discussed in this paper.

\section{MECHANICAL SYSTEM}

The framework of LSTs is basically an enlarged copy of the successfully operating 17m MAGIC telescopes. However, many improvements are introduced with the goal to reduce the costs, increase the remote operability and to limit the maintenance, which are critical issues for big arrays of telescopes as CTA is.

The mechanical structure of the LST telescope follows an alt-azimuth model, and the three main elements are the telescope base, the mount and the optical support structure, as shown in Figure 1.

Big emphasis is given to the low weight, high stiffness and simplicity, because one of the main aims of LST is to quickly react in case of a Gamma Ray Burst (GRB) alert, by changing target from and to any part of the observable sky within few tens of seconds. In fact, the design choice foresees a lightweight tubular structure with a significant use of carbon fiber technology, which allows a considerable reduction on the weight of LST while achieving a high stiffness.

\subsection{Azimuth Lower Structure and Assembly}

The objective of the azimuth moving system of the LST is to allow the telescope to turn along its vertical axis for repositioning and tracking. The system also has to hold the telescope in place under strong storm conditions. During strong storms, the telescope experiences uplift forces which have to be held by the bogies and the rails mounted on the foundation. 

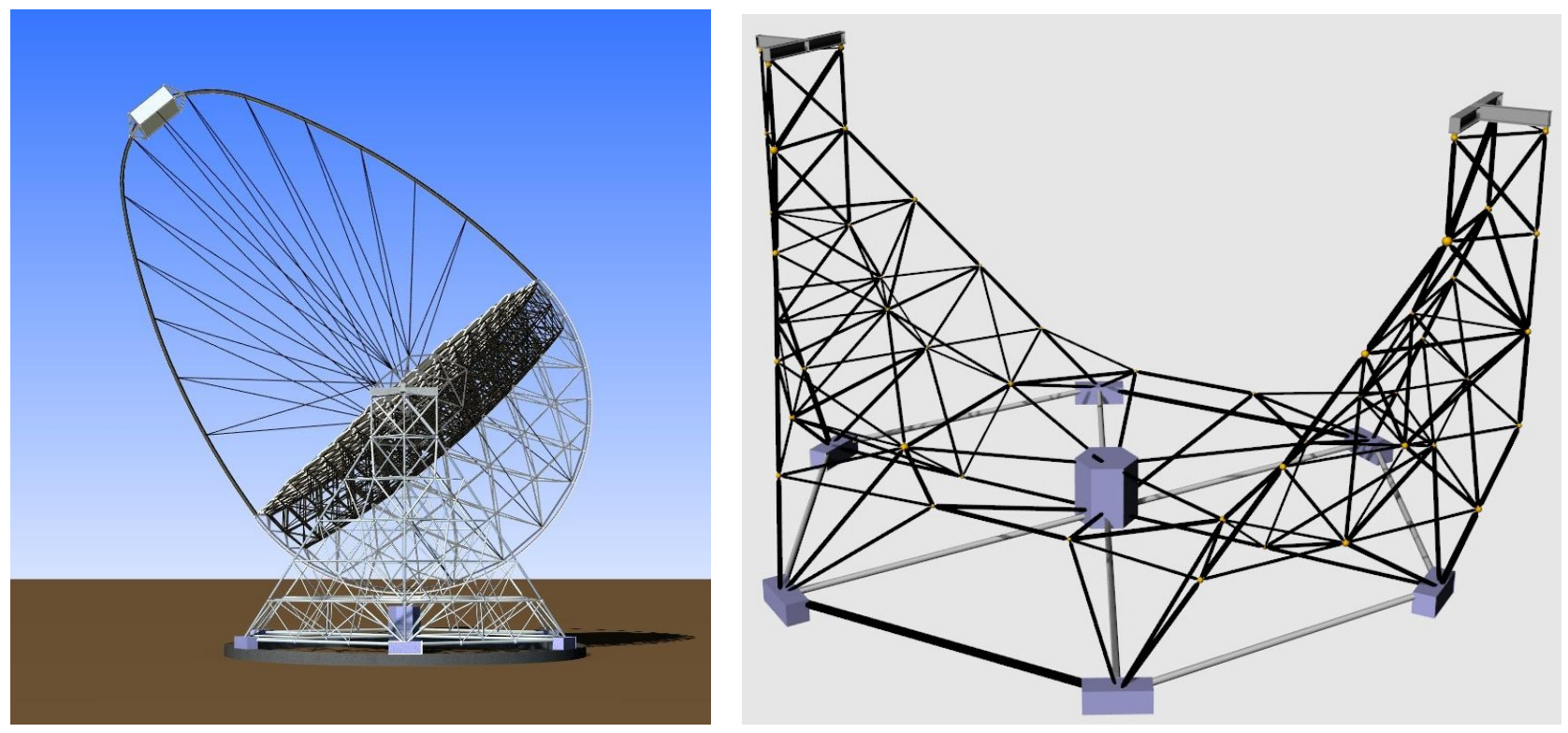

Figure 1. A rendered picture (left) of the telescope's structure and (right) a detailed view of the telescope support structure.

The telescope structure rests on six bogies equally spaced in a hexagonal array, see Figure 1 (right). Two of the bogies are located under the Nasmyth bearings, withstanding the highest fraction of the telescope weight [2].

The flat rail is $50 \mathrm{~cm}$ wide and it is anchored with bolts into the concrete foundation. Each bogie is designed as a modular box with four flat wheels $(34 \mathrm{~cm}$ diameter and $9 \mathrm{~cm}$ width, see Figure 2) running on the top side of the rail while two other flat wheels $(20 \mathrm{~cm}$ diameter) are symmetrically arranged on the lower side of the rail to prevent the bogie from lifting. Four additional similar wheels are mounted on the side walls of the rail to keep the bogie in track.

The azimuth driving system is an inner spur gear (crown) of $24 \mathrm{~m}$ diameter which is installed on the top side of the rail to prevent additional moments onto the bogie during acceleration and deceleration.

Each one of the heavy bogies has two pinions powered by servomotors, turning the telescope. The spur gear assures that the azimuth system always turns, even if the bogie wheels slide. Bogies and rail are covered in order to enlarge the azimuth system lifespan. This cover intends to avoid dust, sand, rocks, ice or snow, pieces, etc pile up on the rail surface increasing the wheel and rail wear. The total bogie weight is $\sim 2500 \mathrm{Kg}$.
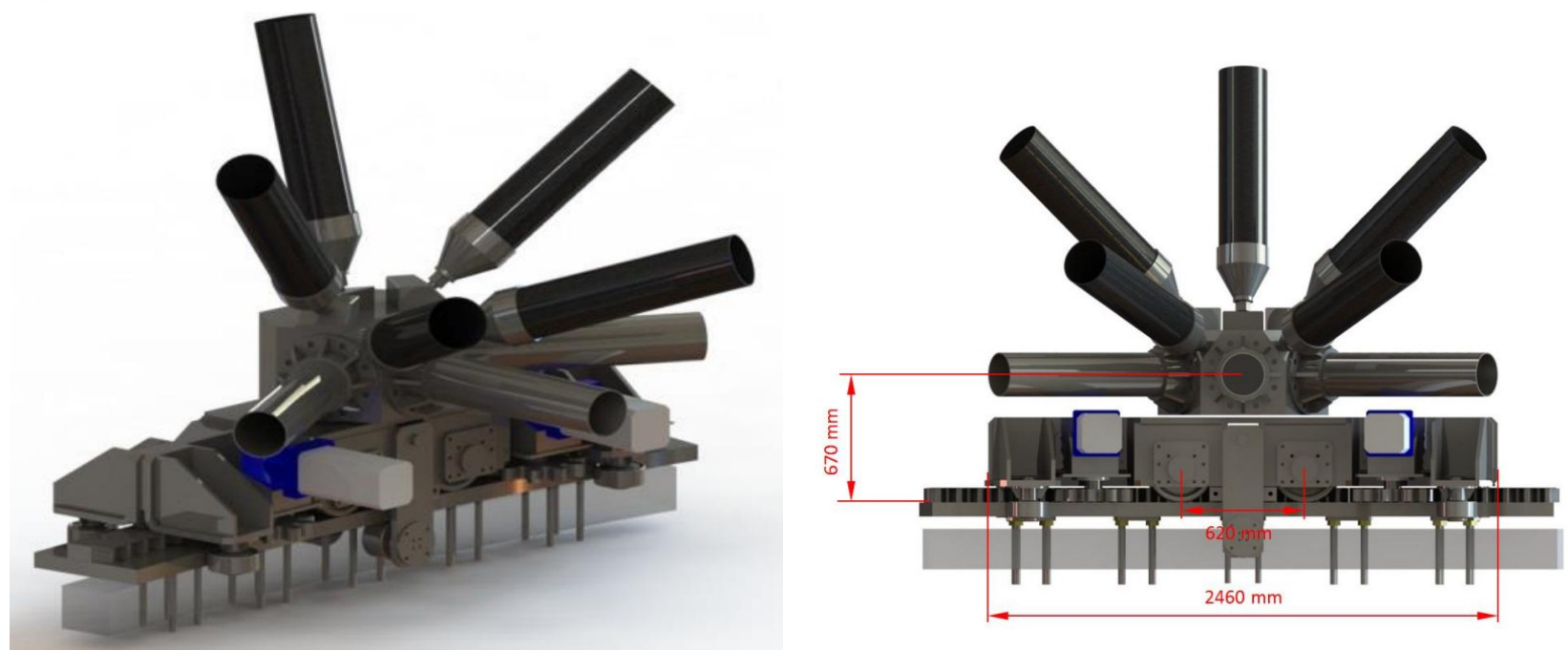

Figure 2. General view of the azimuth driving system (left) and a detail of the bogie design (right). 


\subsection{Elevation Assembly}

The declination drive ring is formed by a curved I-beam acting as a support for a chain bolted ever $5 \mathrm{~cm}$ to the beam. The I-beam has a machined surface which is as a rail for the box carrying the $10 \mathrm{~kW}$ declination drive motor and a disc brake to prevent the dish rotation in case of a motor failure. The motor is connected by a low play planetary gear and a sprocket wheel to the chain thus forming basically a rack and pinion system. The drive box is positioned off-center in order to park the telescope at $95^{\circ}$ from Zenith, but limiting the range in reverse mode to $-70^{\circ}$. The drive box is connected

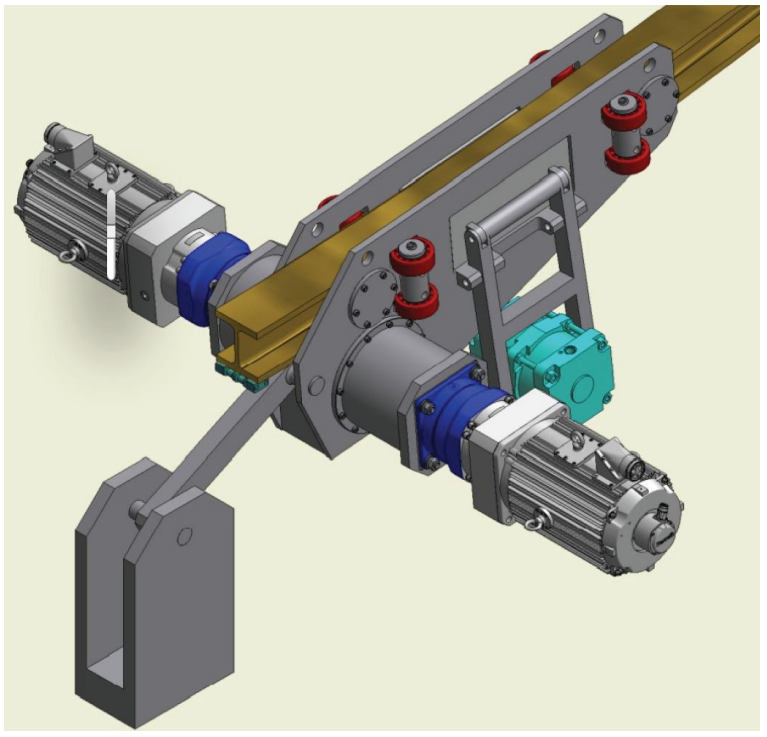

Figure 3. Current design of the elevation drive. to the central axis (king pin) of the telescope by one long arm to compensate for slight changes of the nonperfectly circular drive ring being also not perfectly aligned with the dish axis.

Figure 3 shows the current design of the elevation drive. The drive is mounted slightly asymmetrical; thus it will be possible to park the telescope and to access the camera at 100 degree zenith angle in the park position. The camera will be installed at a height of approximately $10 \mathrm{~m}$ from the ground and has to be accessed by an access tower. The elevation drive is mounted on the circular backside arch of the dish in a way that it tolerates the backside arch to deviate from the perfect circle. The elevation drive is hanging freely on the backside arch and is fixed at the central axis by a supporting beam. There will be an automatic braking and fixation system to fasten the telescope in parking position. This will prevent the telescope to get loose during a storm. A second motor allows movements of the dish if one motor breaks.

\subsection{Drive System}

The fast and precise movement of the LST telescopes will be achieved using electric servomotors on both the elevation and azimuth axis. Four synchronized motors will be used for the Azimuth axis and two for the Elevation axis. Each telescope is considered an independent automation subsystem consisting of its own programmable logic controller, drive, controller and sensors.

Due to the combination of high torque during requirement and high accuracy during tracking, synchronous motors will be used. The main dimensioning of the motors is determined by the fast repositioning ( $20 \mathrm{~s}$ to any position) during a GRB alert, under normal operational loads such as wind. As the duration of the repositioning is short and is not occurring often, it is envisaged to use the motors in overload mode.

The mechanical power peak is estimated to be $145 \mathrm{~kW}$ for the azimuth and $45 \mathrm{~kW}$ for the elevation drive (Figure 3), therefore $12 \mathrm{~kW}$ nominal power motors will used. All the components are based on standard and reliable industrial solutions. The selected family of motors (1FT7 from Siemens) has a $4 \times$ overload capacity during short periods of time.

An energy storage system has been already considered in case the site power network cannot easily provide the required power peak. The solution is based in the usage of flywheels as both the needed power and time would fit with standard industrial solutions.

The other important hardware security must protect the telescope against the climatic conditions and especially the wind. Constant communication with the weather station is foreseen. With no response the telescope goes automatically to its park position. 


\section{OPTICAL SYSTEM}

The $23 \mathrm{~m}$ diameter reflector of LST is a segmented mirror consisting of hexagonal facets that measure $1510 \mathrm{~mm}$ between flat edges. Each facet has a spherical profile with a specific focal length that depends on its position in the reflective surface. The mirror facets are mounted in the nodes of the telescope dish and they are arranged in a way that the reflector gets a parabolic profile to minimize additional time spread of the very fast Cherenkov light flash, see Figure 4.

In total the dish surface can accommodate 207 reflective units. However, six segments at the edge of the dish (four in the sides and two in the bottom) are found to be interfered with the space frame of the mechanical structure. The center of the reflector will not be installed with mirrors because of the camera shadow but it will be equipped with calibration instrumentation that will be mounted on the same supporting rods used for the mirror installation.

Finally, 200 mirror facets at most can be installed, the total reflective area taking into account shadows of the mechanical structures (e.g., the camera photo-sensors, mast, etc...) is about $368 \mathrm{~m}^{2}$.
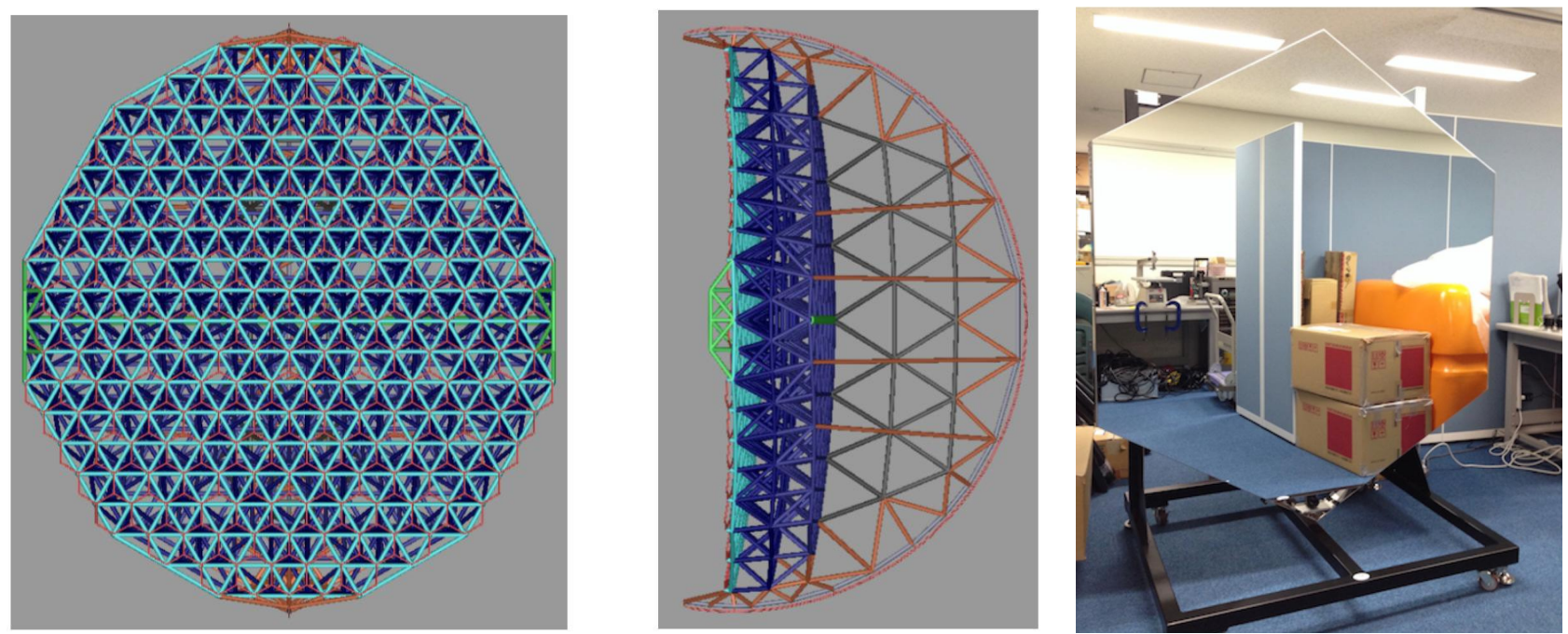

Figure 4. Two views on the LST dish, from the front (left) and the side (center), the light blue elements are the mirror supporting rods. No mirrors are shown. A $1.5 \mathrm{~m}$ mirror produced by Sanko company, Japan (right).

The mirror tiles are supported by a large space frame made from high strength carbon fiber (CF) reinforced tubes. In order to correct small deformations of the mirror due to sagging of the support frame, each mirror tile is corrected by the so-called Active Mirror Control (AMC) similar to the version used in MAGIC but now operating in real time.

The mirrors are manufactured using the cold slump technique [3] and fabricated by the Japanese company Sanko with a sandwich structure consisting of two glass sheets that include an aluminum honeycomb box, see Figure 4 (right).

\section{CAMERA}

The LST camera of 2.0 tons weight will be supported by a single arch camera support mast construction [4], similar to the construction of the MAGIC telescope design, see Figure 5 (left). The parabolic shaped mast will be made from a curved tubular $\mathrm{CF}$ construction of a $30 \mathrm{~cm}$ diameter tube. Transverse stability will be enforced by a number of high strength CF-ropes (of the same kind as used for sailing boats or in oil industry). The camera is located $28 \mathrm{~m}$ above the mirror (reference mirror center).

The camera has to provide protection to all its components, be air-tight and the total weight kept below $2000 \mathrm{~kg}$. A system to load and unload the camera from the camera frame onto the access tower for maintenance is provided.

The camera body relies in a simple tubular structural element which holds all the camera elements and provides the necessary stiffness. The camera skin is made by an aluminum honeycomb shell which offers the best compromise between stiffness and lightness. The skin is coated with a white $\mathrm{TiO}_{2}$ paint that provides protection from insulation. 
The front part of the camera body, which is closed by a Plexiglass window, hosts the light guides coupled to the clusters of photo-multipliers. The rear part accommodates the auxiliary mechanics and electronics, and it is closed by a special cover which assures a perfect sealing.
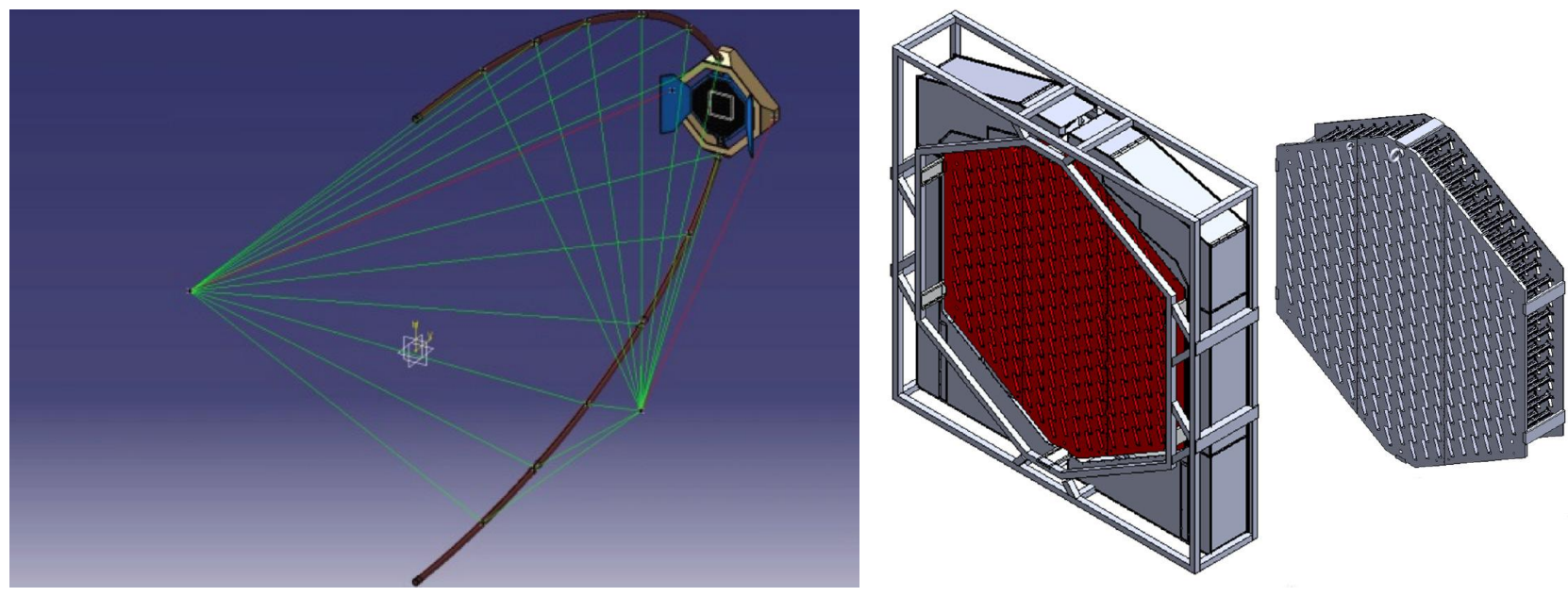

Figure 5. The camera supporting arch (left) and camera body (right).

The camera is built around the cluster concept, a modular unit that contains all the key components of the detector: light guides, photo-sensors and front-end electronics. The mechanical structure is designed to accommodate those camera modules and to allow their easy installation and removal.

The electronics contained in the camera case will dissipate more than $6 \mathrm{~kW}$ during operation. In order to keep the component temperature in the working range a mixed air-liquid cooling has been designed. A water-cooling-heat exchanger is located in the cluster holder and air is circulated in the cluster holder by several funs. The coolant water transfers the heat to a chiller which is mounted on the basement of the telescope. Also the coolant water is circulated in the front plate of the cluster holder which works as a cooling plate as well. The camera clusters are thermodynamically connected to this cooling plate. See [5] for more details.
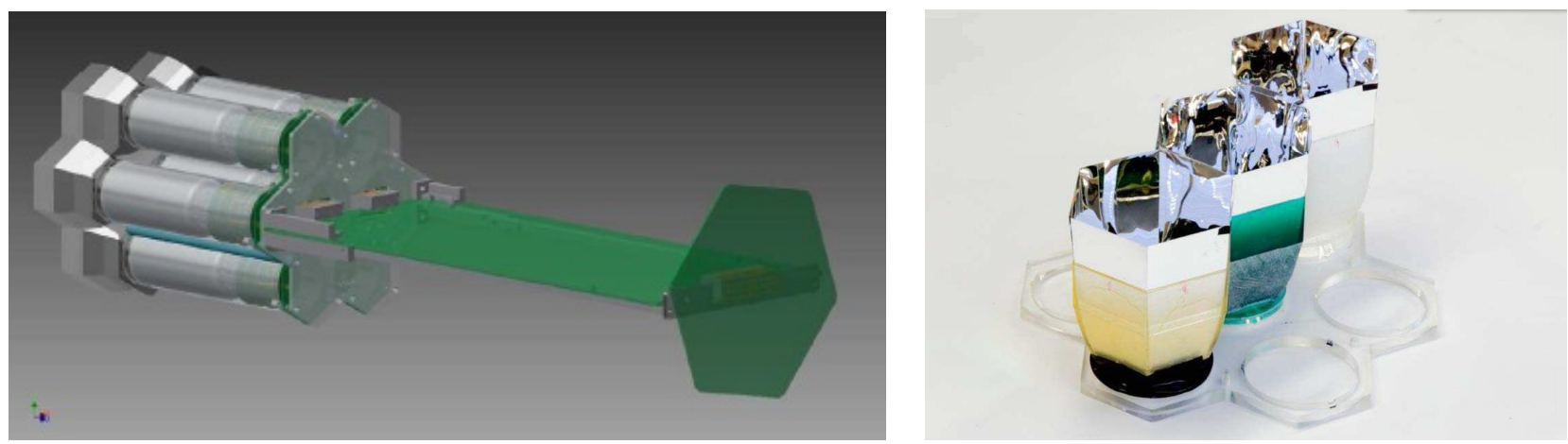

Figure 6. Image of a cluster (left) and light guide (right)

\subsection{Cluster}

The cluster is composed of 7 photomultiplier (PMT) modules and a read-out board as shown in Figure 6 (left). The cluster is a basic unit of data acquisition system including trigger and communications. For a detailed description see [6]. The read-out board is connected to a back-plane board which communicates with other clusters, central control, and slow control system of the camera as well as providing DC power to the read-out board.

To match the pixel light entrance to the PMT photocathode a hexagonal shape light guide is attached on each PMT. 
The light path optimization is based on a ray trace simulation [7], the reflectivity of the light guide is around $90 \%$ for UV light, depending on its incident angle.

The light sensors in the focal plane of the camera must detect the Cherenkov photons generated by air showers in the atmosphere. The Cherenkov light spectrum presents a cut-off around $\sim 300 \mathrm{~nm}$ due to absorption by the oxygen and ozone, and towards longer wavelengths its intensity falls off as $1 / \lambda^{2}$. Beyond $\sim 650 \mathrm{~nm}$, the signal to noise ratio degrades because of the increasing intensity of the night sky background. It is therefore mandatory to select a light sensor which has a high sensitivity in the domain $300 \div 650 \mathrm{~nm}$.

The selected light sensor, baseline for the LST camera, is the Hamamatsu R11920-100-20, see Figure 7. It features:

- $\quad$ eight dynodes design

- operating high voltage $\sim 1 \mathrm{kV}$

- peak sensitivity $40 \%$ at $340 \div 360 \mathrm{~nm}$

The PMT is equipped with the HV power supply electronics based on a Cockcroft-Walton design with a stabilized photo-cathode to first dynode voltage of $300 \mathrm{~V}$ by using a Zener diode. The generated HV is typically thousand times larger than the low voltage and it can be programmed between $850 \mathrm{~V}$ and $1500 \mathrm{~V}$. The HV stability, as well as the ripple, are less than $1 \mathrm{~V}$. The design has been optimized for low consumption applications and it dissipates only $35 \mathrm{~mW}$ of heat.
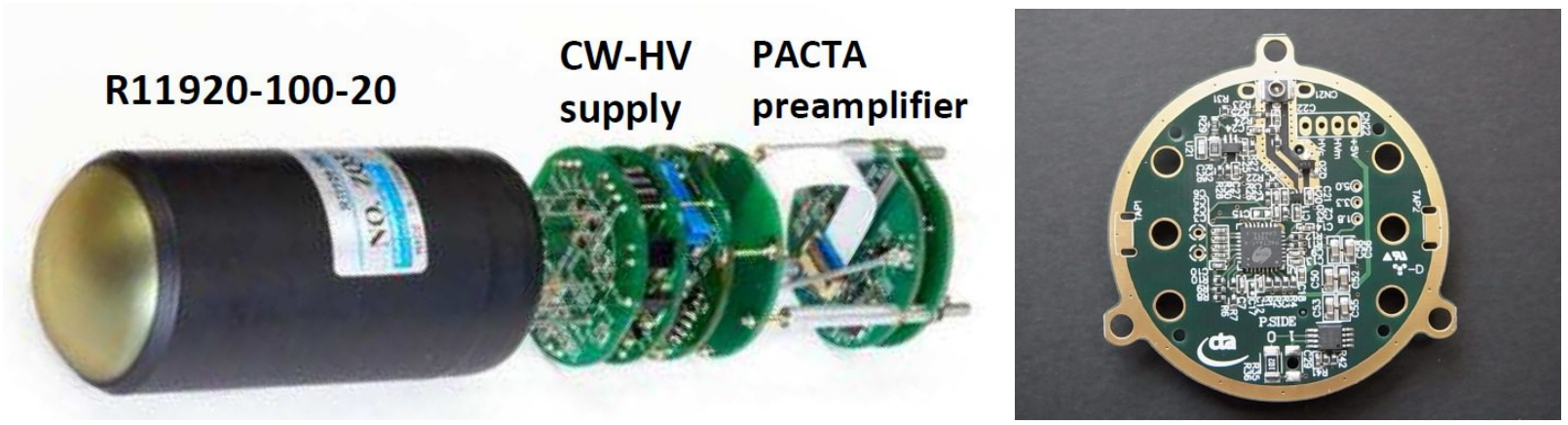

Figure 7. A picture of the PMT assembly, showing the Hamamatsu R11920-100-20, high voltage power supply and preamplification circuit (left). Detail of the preamplification circuit showing the PACTA preamplifier (right).

The analog signal from the PMT is transferred through a co-axial cable with $50 \Omega$ impedance to a preamplifier board. A percentage of 100,50 or $30 \%$ of the signal go through the divider circuit and remaining signal is discarded into the ground line.

In other words, the gain of PMT times that of the divider circuit is adjusted to be 40000 . Then the signal is multiplied by a preamplifier ASIC, called PACTA [8], which is a two-gain transimpedance amplifier with gains of $\times 24$ and $\times 1.6$.

The signals are transmitted to a read-out board so called DRAGON, see Figure 8. An interface board, so called Slow Control Board, is located between the PMT modules and the read-out board to provide power to the PMT modules, monitor HV and anode current, read temperature sensors etc.

The analog signals are divided into three branches: a high gain sent to the trigger system [9], a high and low gain sent to analog samplers to record the signal waveform. The bandwidths of the high and low gain channels are $300 \mathrm{and} 190 \mathrm{MHz}$ respectively. The analog signals are sampled by the Domino Ring Sampler ver.4 (DRS4) ASICs [10]. By exploiting the DRS4 configurability, each signals is recorded by a ring of 4096 storage cells that, at a sampling speed of 1 GSPS, corresponds to a total depth of $\sim 4 \mu \mathrm{s}$.

An external signal provided by the trigger system starts the signal digitization by means of an external A/D converter and the data are transmitted the data acquisition system by a Gigabit Ethernet transceiver as well as to the camera trigger system, both located on a backplane. For more details see [11]. 


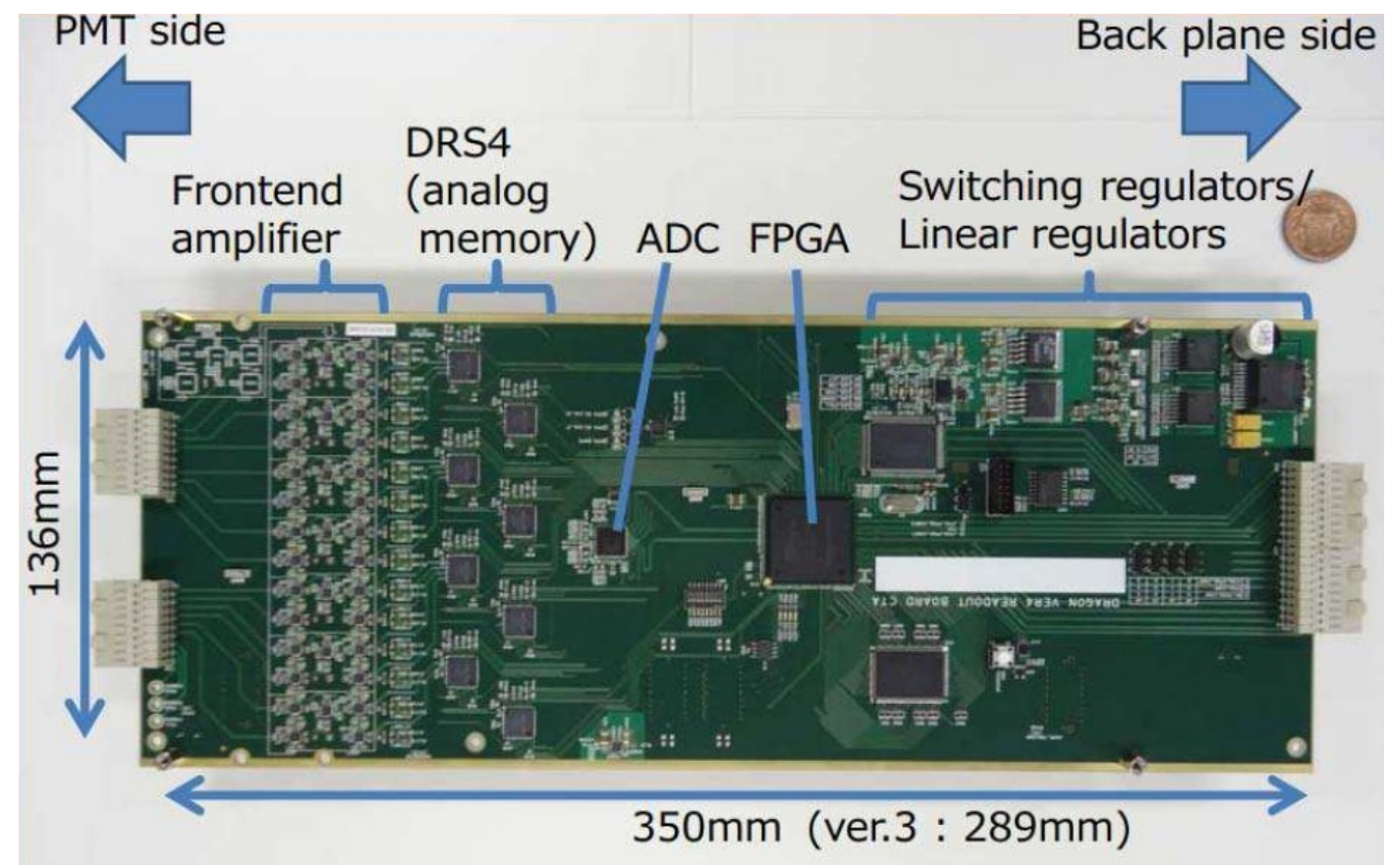

Figure 8. A picture of the Dragon board: from left to right is the amplification, sampling, digitization and FPGA.

\section{ACKNOWLEDGMENTS}

We gratefully acknowledge financial support from the following agencies and organizations: Ministerio de Ciencia, Tecnología e Innovación Productiva (MinCyT), Comisión Nacional de Energía Atómica (CNEA), Consejo Nacional de Investigaciones Científicas y Técnicas (CONICET), Argentina; State Committee of Science of Armenia, Armenia; Conselho Nacional de Desenvolvimento Científico e Tecnológico (CNPq), Fundação de Amparo à Pesquisa do Estado do Rio de Janeiro (FAPERJ), Fundação de Amparo à Pesquisa do Estado de São Paulo (FAPESP), Brasil; Croatian Science Foundation, Croatia; Ministry of Education, Youth and Sports, MEYS LE13012, 7AMB12AR013, Czech Republic; Ministry of Higher Education and Research, CNRSINSU and CNRS-IN2P3, CEA-Irfu, ANR, Regional Council Ile de France, Labex ENIGMASS, OSUG2020 and OCEVU, France; Max Planck Society, BMBF, DESY, Helmholtz Association, Germany; Department of Atomic Energy, Department of Science and Technology, India; Istituto Nazionale di Astrofisica (INAF), MIUR, Italy; Istituto Nazionale di Fisica Nucleare (INFN), Italy; ICRR, University of Tokyo, JSPS, Japan; Netherlands Research School for Astronomy (NOVA), Netherlands Organization for Scientific Research (NWO), Netherlands; The Bergen Research Foundation, Norway; Ministry of Science and Higher Education, the National Centre for Research and Development and the National Science Centre, Poland; MINECO support through the National R+D+I, CDTI funding plans and the CPAN and MultiDark Consolider-Ingenio 2010 programme, Spain; Swedish Research Council, Royal Swedish Academy of Sciences, Sweden; Swiss National Science Foundation (SNSF), Ernest Boninchi Foundation, Switzerland; Durham University, Leverhulme Trust, Liverpool University, University of Leicester, University of Oxford, Royal Society, Science and Technologies Facilities Council, UK; U.S. National Science Foundation, U.S. Department of Energy, Argonne National Laboratory, Barnard College, University of California, University of Chicago, Columbia University, Georgia Institute of Technology, Institute for Nuclear and Particle Astrophysics (INPACMRPI program), Iowa State University, Washington University McDonnell Center for the Space Sciences, USA.

The research leading to these results has received funding from the European Union's Seventh Framework Programme (FP7/2007-2013) under grant agreement n 262053. 


\section{REFERENCES}

[1] The Cherenkov Telescope Array, http://www.cta-observatory.org/

[2] Cortina J., et al., "The Large Size Telescope, a $23 \mathrm{~m}$ diameter Cherenkov telescope for the Cherenkov Telescope Array made of high strength, low weight carbon fiber reinforced plastic tubes," Proc. ICRC, (2013)

[3] Vernani D., et al., "Glass mirrors by cold slumping to cover $100 \mathrm{~m}^{2}$ of the MAGIC II Cherenkov telescope reflecting surface," Proc. SPIE 7018, (2008)

[4] Lamanna G., et al., "Large Size Telescope camera support structures for the Cherenkov Telescope Array," Proc. ICRC, (2013)

[5] Delgado C., et al., Mechanics and cooling system for the camera of the Large Size Telescopes of the Cherenkov Telescope Array (CTA)," Proc ICRC, (2013)

[6] Yamamoto T., "Development of the Camera for the Large Size Telescopes of the Cherenkov Telescope Array," Proc. SPIE, (2014).

[7] Okumura A., https://github.com/akira-okumura/ROBAST

[8] Sanuy A. et al., "Wideband (500 MHz) 16 bit dynamic range current mode PreAmplifier for the CTA cameras (PACTA)," JINST, 7, (2012)

[9] Tejedor L.A., et al., "An Analog Trigger System for Atmospheric Cherenkov Telescope Arrays," IEEE Transactions on Nuclear Science, Vol. 60 Issue 3, (2013)

[10] Sulanke K.H., et al., " A Digital Camera Trigger for the Cherenkov Telescope Array," Proc. ICRC, (2013)

[11]Ritt S., Dinapoli R., Hartmann U., " Application of the DRS chip for fast waveform digitizing," Nuclear Instruments and Methods A Vol. 623, (2010)

[12] Kubo H., Paoletti R., et al., "Development of the Photomultiplier-Tube Readout System for the CTA Large Size Telescope," Proc. ICRC, (2013) 